\title{
Magnetospheric accretion-ejection processes in the classical T Tauri star AA Tauri ${ }^{\star}, \star \star$
}

\author{
J. Bouvier ${ }^{1}$, S. H. P. Alencar ${ }^{1,2}$, T. Boutelier ${ }^{1}$, C. Dougados ${ }^{1}$, Z. Balog ${ }^{3,4}$, K. Grankin ${ }^{5}$, \\ S. T. Hodgkin ${ }^{6}$, M. A. Ibrahimov ${ }^{5}$, M. Kun ${ }^{7}$, T. Yu. Magakian ${ }^{8}$, and C. Pinte ${ }^{1}$ \\ 1 Laboratoire d'Astrophysique de Grenoble, Université Joseph-Fourier, BP 53, 38041 Grenoble Cedex 9, France \\ e-mail: jbouvier@obs.ujf-grenoble.fr \\ 2 Departamento de Física, ICEx-UFMG, CP. 702, Belo Horizonte, MG, 30123-970, Brazil \\ 3 Steward Observatory, University of Arizona, 933 N. Cherry Av., Tucson AZ 85721, USA \\ 4 Department of Optics and Quantum Electronics, University of Szeged, Dóm tér 9, 6720 Szeged, Hungary \\ 5 Ulugh Beg Astronomical Institute of the Uzbek Academy of Sciences, Astronomicheskaya 33, 700052 Tashkent, Uzbekistan \\ ${ }^{6}$ Institute of Astronomy, Madingley Road, Cambridge CB3 0HA, UK \\ Konkoly Observatory, 1525 Budapest PO Box 67, Hungary \\ 8 V.A. Ambartsumyan Byurakan Astrophysical Observatory, Armenia
}

Received 12 July 2006 / Accepted 28 November 2006

\section{ABSTRACT}

\begin{abstract}
Context. Accretion and ejection are complex and related processes that vary on various timescales in young stars. Aims. We intend to investigate the accretion and outflow dynamics and their interaction from observations of the classical $\mathrm{T}$ Tauri star AA Tau.

Methods. From a long time series of high resolution $(R=115000)$ HARPS spectra and simultaneous broad-band photometry, we report new evidence for magnetospheric accretion as well as ejection processes in the nearly edge-on classical T Tauri star AA Tau. Results. AA Tau's light curve is modulated with a period of $8.22 \mathrm{~d}$. The recurrent luminosity dips are due to the periodic occultation of the central star by the magnetically-warped inner disk edge located at about $9 R_{\star}$. Balmer line profiles exhibit a clear rotational modulation of high-velocity redshifted absorption components with a period of 8.22 days as well, with a maximum strength when the main accretion funnel flow passes through the line of sight. At the same time, the luminosity of the system decreases by about $1 \mathrm{mag}$, indicative of circumstellar absorption of the stellar photosphere by the magnetically-warped, corotating inner disk edge. The photospheric and $\mathrm{He}$ I radial velocities also exhibit periodic variations, and the veiling is modulated by the appearance of the accretion shock at the bottom of the accretion funnel. Diagnostics of hot winds and their temporal behaviour are also presented.

Conclusions. The peculiar geometry of the young AA Tau system (nearly edge-on) allows us to uniquely probe the acretion-ejection region close to the star. We find that most spectral and photometric diagnostics vary as expected from models of magneticallychannelled accretion in young stars, with a large scale magnetosphere tilted by $20^{\circ}$ onto the star's spin axis. We also find evidence for time variability of the magnetospheric accretion flow on a timescale of a few rotational periods.
\end{abstract}

Key words. accretion, accretion disks - stars: pre-main sequence - stars: magnetic fields - stars: individual: AA Tau

\section{Introduction}

For a few million years after their formation, young solar-type stars (T Tauri stars, TTS) continue to accrete material from their circumstellar disks. The accretion process has a profound and long lasting impact on early stellar evolution. It is thought to be the driving source of the powerful jets originating from young stars and to regulate their angular momentum before they reach the zero age main sequence. Accretion is also the central ingredient of disk evolution at a time when planets start to form, and accretion both into the disk and onto the star is held responsible for most of the peculiar properties of $\mathrm{T}$ Tauri stars (see, e.g., the review by Ménard \& Bertout 1999). Understanding how

* Based on observations obtained at ESO Obs., Chile, Maidanak Obs., Uzbekistan, Buryakan Obs., Armenia, Konkoly Obs., Hungary, and La Palma Obs., Spain.

$\star \star$ Photometric data are only available in electronic form at the CDS via anonymous ftp to cdsarc.u-strasbg. fr (130.79.128.5) or via http://cdsweb.u-strasbg.fr/cgi-bin/qcat?]/A+A/463/1017 accretion proceeds into the disk and onto the star is therefore a major issue for star and planet formation theories.

It is now commonly acknowledged that accretion from the inner disk onto the star is, at least in part, mediated by the stellar magnetic field. Recent Zeeman measurements indicate surface magnetic fields of a few kilogauss in T Tauri stars (e.g. Johns-Krull et al. 1999, 2004; Symington et al. 2005a), strong enough to disrupt the inner disk region and control the accretion flow. It is thus expected that the inner disk edge lies at a few stellar radii from the central star and that material is accreted onto the star along funnel flows which follow the topology of the large scale component of the stellar magnetosphere. As the freefalling material eventually reaches the star, the kinetic energy is dissipated in a shock at the stellar surface (see, e.g. Königl 1991).

Recent numerical simulations of the magnetic star-disk interface confirm most of these expectations. Assuming a dipolar magnetosphere on the large scale, whose axis can either be aligned with or inclined onto the stellar rotation axis, simulations show the development of the inner disk truncation by strong 
Table 1. Journal of photometric observations.

\begin{tabular}{llllll}
\hline \hline Observatory & JD-2 450000 & Filters & Observer & Country & $N_{\text {obs }}(V)$ \\
\hline Maidanak & $3260 .-3311$. & Tube $B V$ & K. Grankin & Uzbekistan & 19 \\
Maidanak & $3307 .-3346$. & CCD $B V$ & M. Ibrahimov & Uzbekistan & 32 \\
Buryakan & $3297 .-3316$ & CCD $B V$ & T. Magakian & Armenia & 5 \\
Konkoly & $3334 .-3382$ & CCD $B V$ & Z. Balog & Hungary & 32 \\
IAC & $3326 .-3396$ & CCD $V$ & S. Hodgkin & Canarias & 28 \\
\hline
\end{tabular}

magnetic fields, leading to a magnetospheric cavity extending over a few stellar radii (e.g. Romanova et al. 2002, 2003). In this magnetically-controlled region, material is accreted onto the star through a complex and time variable network of funnel flows, whose structure and evolution depend on the accretion rate, on the topology of the magnetosphere and its inclination on the stellar axis (e.g. Romanova et al. 2003). As a result, the accretion shock at the stellar surface can take various shapes and be located at different latitudes (Romanova et al. 2004). Both numerical simulations and analytical models suggest that the inner disk structure is significantly modified by its interaction with the stellar magnetosphere (Lai 1999; Terquem \& Papaloizou 2000).

The winds of classical T Tauri stars (CTTSs) have been primarily investigated through the observations and modelling of forbidden emission lines, whose high velocity component originates in spatially extended microjets (Hartigan et al. 1995; Shang et al. 2002). A few CTTSs had actually their microjets emission imaged. They arise in a region very close to the star and were observed with ground based adaptive optics (Dougados et al. 2000) and HST (Bacciotti et al. 2002), providing unprecedent information on the dynamics near the base of the jet, where the wind is formed. Other high velocity inner disk wind diagnostics include cooler outflowing gas that absorbs emission from the accreting material (Edwards 2003) and the observed hot helium gas in emission (Beristain et al. 2001) as well as P Cygni profiles with strong blueshifted absorptions reaching well into the stellar continuum over a broad velocity range (Edwards et al. 2003; Dupree et al. 2005). The above wind signatures are prominent in CTTSs with high mass accretion rates, indicating that these are actually accretion-powered winds.

With an extension of a few stellar radii up to the inner disk edge $(\simeq 0.05 \mathrm{AU})$, the magnetospheric cavity can hardly be resolved directly, even with the most powerful interferometers (e.g. Akeson et al. 2005). In addition, the magnetospheric cavity is filled with hot gas while dust particules are likely sublimated. Hence, diagnotics of hot plasma would have to be observed at this scale, which requires interferometric observations at high spectral resolution. For the time being, only indirect signatures of the structure of the star-disk interaction region and its physical properties can therefore be searched for.

As the magnetospheric cavity up to the disk inner edge is thought to corotate with the central star, a powerful approach to confront model predictions is to monitor the variations of suited photometric and spectral diagnostics over a few rotational periods. Synoptic studies may reveal periodic variability which can then be associated to physical structures in the rotating magnetosphere and the inner disk. A number of such studies have been performed in the last decade providing (sometimes conflicting) clues to the magnetospheric accretion process in young stars (see Bouvier et al. 2007, for a review).

Two of our previous monitoring campaigns (Bouvier et al. 1999, 2003, Papers I and II hereafter) have focused on the classical T Tauri star AA Tau. Thanks to its high viewing angle $\left(i \simeq 75^{\circ}\right)$, the line of sight to the stellar surface intercepts the star-disk interface close to the disk plane. This fortunate configuration maximizes the observational signatures of the magnetospheric accretion process. Furthermore, AA Tau is quite a typical classical T Tauri star, with a K7 spectral type, moderate accretion rate and Class II SED. Hence, it is likely that the results obtained for this otherwise unremarkable CTTS can be extended to the whole class of CTTSs.

From our previous campaigns on AA Tau we reported evidence for the modulation of photometric and spectroscopic diagnostics with a period of 8.2 days, the rotational period of the star. The modulation of the stellar flux was interpreted as resulting from the periodic occultation of the stellar photosphere by the optically thick, magnetically-warped inner disk edge located at 8.8 stellar radii (Bouvier et al. 1999). A second campaign allowed us to relate the recurrent eclipses of the star by the inner disk to accretion funnel flows and accretion shocks at the stellar surface (Bouvier et al. 2003). We also found evidence for large scale changes occurring in the accretion flow onto the star on a timescale of a few weeks, presumably resulting from a major disturbance of the underlying magnetospheric structure.

In this paper, we report the results of a new campaign on AA Tau aimed at further constraining the structure of the magnetically-dominated star-disk interaction region. We have monitored the photometric variations of the system over nearly 5 months in the fall of 2004, and during part of the photometric campaign, we simultaneously obtained a series of high resolution $(R=115000)$ spectra on the object. To our knowledge, this is the first time such a series of high resolution spectra have been obtained on a T Tauri star. In Sect. 2, we describe the photometric and spectroscopic observations obtained during this campaign. In Sect. 3, we present the results on the photometric and spectroscopic variability of AA Tau, which we find to be periodic with the same period as reported previously. In Sect. 4, we discuss the new evidence provided by these observations for magnetospheric accretion and ejection processes in this object.

\section{Observations}

\subsection{Photometry}

The Journal of photometric observations is given in Table 1. Observations were carried out from several sites over a time span of nearly 140 days from September 2004 to January 2005, using either CCD detectors or photomultiplier tubes. Measurements were obtained in the $B$ and $V$ filters. Differential photometry was performed on CCD images and absolute photometry from photomultiplier observations, with an accuracy of the order of $0.01 \mathrm{mag}$ in both filters. Somewhat larger systematic errors $(\leq 0.05 \mathrm{mag})$ might result from the relative calibration of the photometry between sites. All data reduction procedures were previously described in Bouvier et al. (2003). The catalogue of photometric measurements is available at the CDS. 
Table 2. Veiling values, photospheric, $\mathrm{He} \mathrm{I}$ and $\mathrm{H} \alpha$ blue and red absorption radial velocities, and equivalent widths of the main emission lines. All radial velocities are expressed in the stellar rest frame $\left(V_{\mathrm{lsr}}=V_{\mathrm{rad}}-17.3 \mathrm{~km} \mathrm{~s}^{-1}\right)$.

\begin{tabular}{llcllllll}
\hline \hline $\begin{array}{l}\text { JD } \\
-2450000 .\end{array}$ & Veiling & $\begin{array}{l}V_{\text {lsr }} \text { phot. } \\
\left(\mathrm{km} \mathrm{s}^{-1}\right)\end{array}$ & $\begin{array}{l}V_{\text {lsr }} \mathrm{He} \mathrm{I} \\
\left(\mathrm{km} \mathrm{s}^{-1}\right)\end{array}$ & $\begin{array}{l}V_{\text {lsr }}(\mathrm{H} \alpha \text { blue }) \\
\left(\mathrm{km} \mathrm{s}^{-1}\right)\end{array}$ & $\begin{array}{l}V_{\text {lsr }}(\mathrm{H} \alpha \mathrm{red}) \\
\left(\mathrm{km} \mathrm{s}^{-1}\right)\end{array}$ & $\begin{array}{l}\mathrm{H} \alpha E W \\
(\AA)\end{array}$ & $\begin{array}{l}\mathrm{He} \mathrm{I} E W \\
(\AA)\end{array}$ & $\begin{array}{l}\mathrm{H} \beta E W \\
(\AA)\end{array}$ \\
\hline 3288.82 & 0.17 & 0.8 & 5.4 & -42.5 & 5.2 & 15.6 & 0.3 & 1.7 \\
3289.82 & 0.26 & 0.7 & 4.0 & -22.7 & 32.2 & 12.3 & 0.7 & 1.4 \\
3291.80 & 0.54 & -3.1 & 5.8 & -24.5 & 30.9 & 21.9 & 1.1 & 2.2 \\
3295.80 & 0.15 & -0.3 & 9.7 & -27.2 & 16.0 & 9.5 & 0.2 & 1.3 \\
3296.81 & 0.15 & 0.7 & 9.6 & -37.3 & 4.9 & 15.1 & 0.3 & 2.2 \\
3297.80 & 0.19 & 0.7 & 4.5 & -26.7 & 16.7 & 15.9 & 0.4 & 1.8 \\
3298.84 & 0.22 & 0.9 & 5.3 & -30.9 & 26.9 & 11.8 & 0.7 & 1.2 \\
3308.77 & 0.18 & -0.4 & 8.2 & - & - & 5.0 & 0.5 & 0.9 \\
3309.81 & 0.31 & -1.0 & 5.2 & - & - & 8.9 & 0.3 & 0.8 \\
3310.78 & 0.21 & -1.0 & 12.2 & -24.2 & 17.5 & 6.7 & 0.4 & 0.7 \\
3311.76 & 0.14 & -1.0 & 6.1 & -27.7 & 17.2 & 7.3 & 0.2 & 0.9 \\
3312.77 & 0.19 & -0.4 & - & -35.0 & 20.9 & 8.4 & 0.2 & - \\
3314.75 & 0.15 & 0.5 & 7.2 & - & - & 63.7 & 0.5 & 4.0 \\
3315.75 & 0.18 & 0.3 & 4.6 & - & - & 34.0 & 0.5 & 2.8 \\
3320.79 & 0.13 & 0.5 & 11.0 & -33.8 & 22.5 & 13.9 & 0.3 & 0.8 \\
3332.69 & 0.40 & 0.7 & 7.1 & -22.9 & 40.1 & 30.5 & 1.2 & 2.5 \\
3335.71 & 0.24 & -2.0 & 11.7 & -12.9 & 44.4 & 19.5 & 0.5 & 2.0 \\
3337.76 & 0.20 & 0.5 & 7.3 & -23.5 & 48.0 & 23.7 & 0.4 & 2.0 \\
3338.73 & 0.27 & 1.2 & 3.2 & -18.9 & 47.2 & 23.6 & 0.7 & 1.7 \\
3339.73 & 0.41 & 1.7 & 3.8 & -15.9 & 49.6 & 43.1 & 1.4 & 3.4 \\
3340.72 & 0.68 & 3.7 & 7.7 & -12.6 & 52.4 & 44.1 & 1.6 & 2.6 \\
3341.72 & 0.41 & 1.1 & 13.0 & -13.4 & 50.6 & 38.3 & 1.1 & 2.4 \\
\hline
\end{tabular}

\subsection{Spectroscopy}

The spectroscopic observations of AA Tau were carried out from October 10 to December 02, 2004, at ESO, La Silla. We obtained 22 high-resolution spectra, over 22 non consecutive nights spread between JD 3288 and JD 3341 at the $3.6 \mathrm{~m}$ telescope with the HARPS dual fiber echelle spectrograph (Mayor et al. 2003) covering the $3800 \AA$ to $6900 \AA$ spectral domain at a spectral resolution of $\lambda / \Delta \lambda \approx 115000$. Integration times of 1800 s yielded a $\mathrm{S} / \mathrm{N}$ ratio between 10 and 30 at $600 \mathrm{~nm}$ depending on the object brightness. Quasi-simultaneous photometry was obtained over the period of the spectroscopic observations (JD 3288-3341, see Table 1). The Journal of spectroscopic observations is given in Table 2.

The data was automatically reduced by the HARPS Data Reduction Software. The reduction procedure includes optimal extraction of the orders and flat-fielding, achieved through a tungstene lamp exposure, wavelength calibration with a thorium lamp exposure and the removal of cosmic rays. Cross-correlation functions are also automatically computed. The background sky is not automatically subtracted but it can be done later by the observer.

\section{Results}

\subsection{Photometry}

AA Tau's $V$-band light curve is shown in Figure 1 over nearly 150 days. It is characterized by a nearly constant level of maximum brightness $(V \simeq 12.4)$ with luminosity drops reaching $1.2 \mathrm{mag}$. The general photometric behaviour of AA Tau during this campaign is qualitatively similar to what we had previously reported for similar light curves obtained in 1995 and 1999 (see Bouvier et al. 1999, 2003). Periodogram (Horne \& Baliunas 1986; Roberts et al. 1987) and string-length (Dworetsky 1983) analyses of the $V$ and $B$-band light curves reveal a clear periodic signal with $P=8.22 \pm 0.03$ days, consistent with previous estimates (8.2-8.6 d, see Vrba et al. 1989;
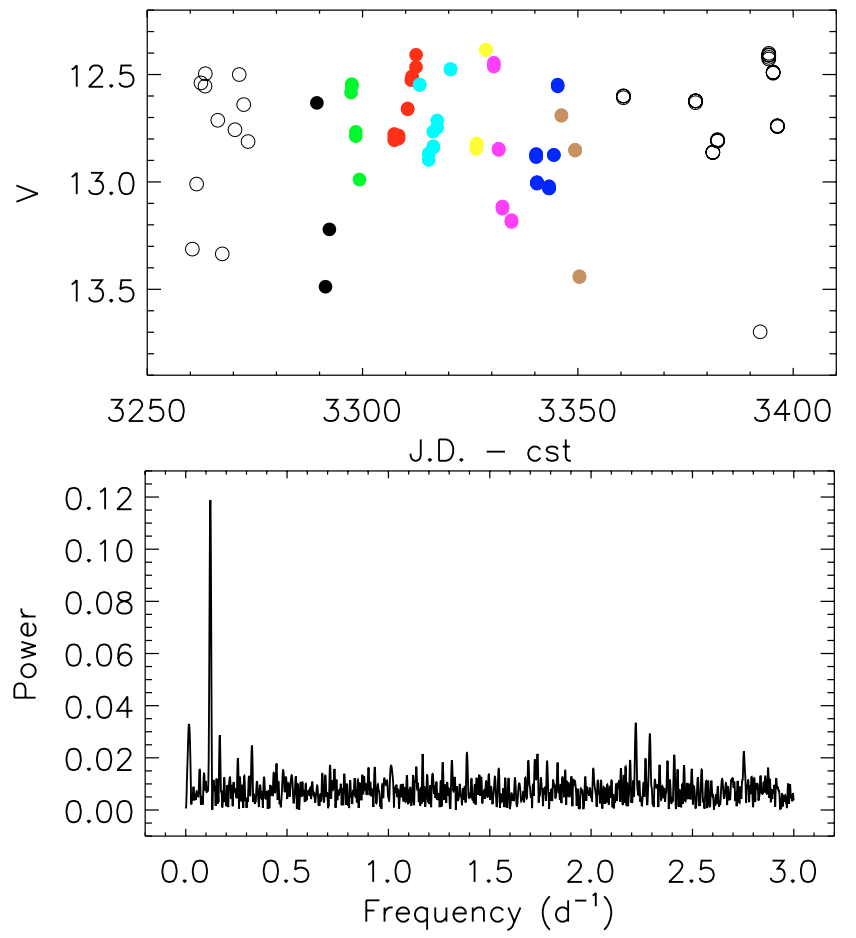

Fig. 1. The $V$-band light curve of AA Tau (top panel) and its CLEANed periodogram (lower panel). $V$-band measurements corresponding to quasi-simultaneous spectroscopic observations were obtained between JD 3285 and 3355 are marked by filled symbols. Different cycles are shown by different colors (or shades of grey).

Shevchenko et al. 1991; Bouvier et al. 1999, 2003). The CLEANed periodogram of the $V$-band light curve is shown in the bottom panel of Fig. 1. The False Alarm Probability (FAP) was computed following the Monte Carlo method described in Herbst et al. (2002). The FAP of the highest periodogram peak was found to be less than $0.01 \%$. The $V$-band light curve folded 


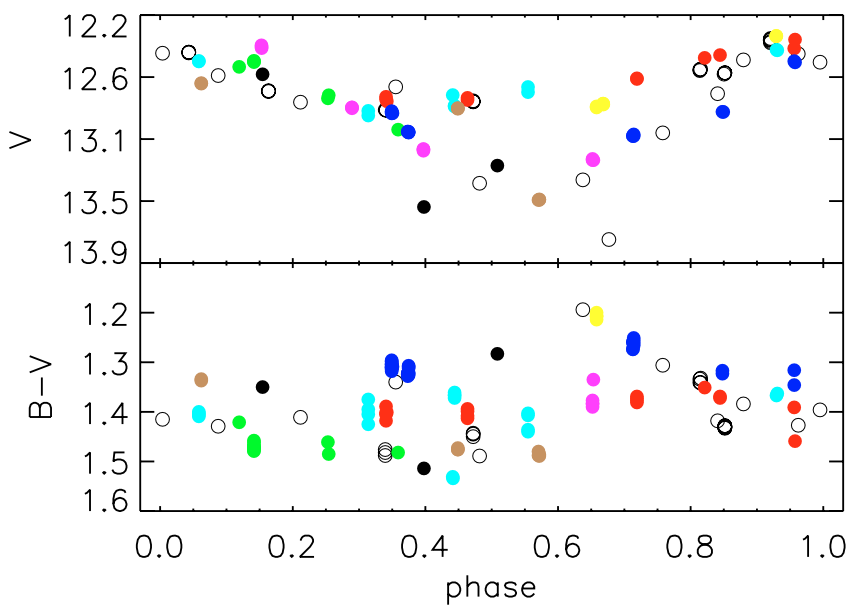

Fig. 2. AA Tau's $V$-band (top) and $B-V$ (bottom) light curves folded in phase with a period of 8.22 days. Different cycles are shown by different colors (or shades of grey).

in phase with this period is shown in Fig. 2. Similar results are obtained for the $B$-band light curve. The phased light curve exhibits a broad brightness minimum with an amplitude of about $1 \mathrm{mag}$. A large dispersion of the flux is seen to occur within the deep minimum.

In previous papers of this series, we argued that the 8.22-day period is the rotational period of the star. From the modelling of AA Tau's spectral energy distribution, Basri \& Bertout (1989) derived an inclination angle of $70^{\circ}$ (see also O'Sullivan et al. 2005). This estimate combined with a $v \sin i$ of $11.3 \mathrm{~km} \mathrm{~s}^{-1}$ (Paper II) and a stellar radius of $1.85 R_{\odot}$ (Paper I), leads to an expected rotational period of $7.8 \mathrm{~d}$ in rough agreement with the measured photometric period. In addition, we report below for the first time a period similar to the 8.2 day photometric period for the flux and radial velocity variations of emission lines as well as for veiling. This supports the 8.2-day period as being the stellar rotation period.

A closer examination of the phased light curve reveals that the amplitude of modulation varies from cycles to cycles, reaching about $1.2 \mathrm{mag}$ from JD 3260 to JD 3300 (5 cycles) and again from JD 3330 to JD 3350 and perhaps later ( $\geq 2.5$ cycles), but amounting to only 0.3 mag between JD 3305 and JD 3330 ( 3 cycles). Even though the amplitude changes, the phase of the modulation appears to be conserved over the whole 140 day-long observations.

$B-V$ colours were computed from each quasi-simultaneous $B$ and $V$ measurements. The phased variations of the $B-V$ colour are shown in Fig. 2. The amplitude of $B-V$ color variations amounts to 0.3 mag only, much shallower than the brightness variations. There is no clear evidence for a modulation of the $B-V$ color within cycles, and most of the dispersion results from $B-V$ color changes from one cycle to the next. It is noteworthy that both the bluest $(\sim 1.3)$ and the reddest $(\sim 1.5) B-V$ colors can occur during the brightness minimum.

In addition to the photometry reported here, we found a number of $V$-band measurements obtained for AA Tau at the same epoch by the All Sky Automated Survey (ASAS, Pojmanski et al. 2005). Adding these measurements to ours, we show the resulting phased light curve in Fig. 3. A periodogram analysis of this dataset confirms the 8.2-day period at the $0.01 \%$ FAP level. ASAS measurements also confirm the stability of the phase of the photometric variations over a timescale of nearly half a year (170 days), while the depth of the photometric minima is much more variable.

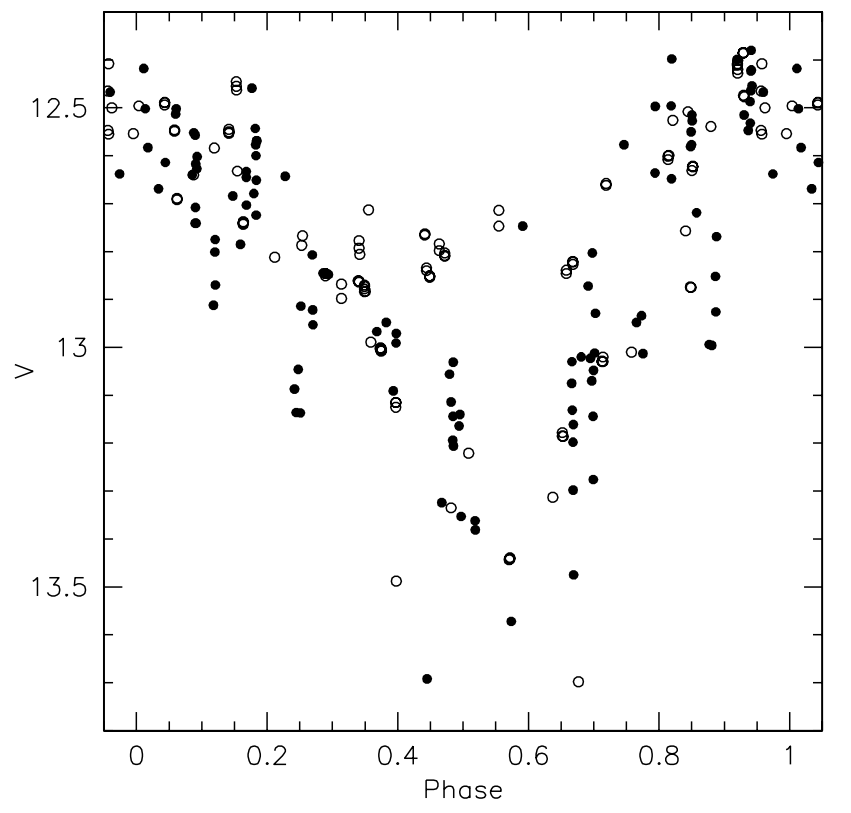

Fig. 3. AA Tau's phased $V$-band light curve $(P=8.22 \mathrm{~d})$ including our own measurements (open dots) as well as ASAS ones (filled dots) covering over more than 5 months, from JD 3230 to JD 3396 . Note the phase stability of the light curve on this time span and the range of variation in the deep brightness minima.

\subsection{Spectroscopy}

\subsubsection{Photospheric lines}

The photospheric lines of CTTS often appear shallower than expected for their spectral types. This is due to an extra continuum, known as veiling, produced at the shock region where the infalling material hits the star, that is added to the stellar continuum. In order to use the veiling as a proxy for accretion onto the stellar surface (cf. Paper I), we computed the veiling using a $\chi^{2}$ fit, based on the method described by Hartigan et al. (1989), on six spectral intervals about $50 \AA$-wide located between $5400 \AA$ and $6700 \AA$. We used as a reference for the veiling calculations a spectrum of the K7 weak T Tauri star V819 Tau obtained with HARPS with the same observing setting as AA Tau. This weak T Tauri star was shown in Paper II to be a very good match to the AA Tau photosphere. The spectrum of V819 Tau was rotationally broadened to the AA Tau $v \sin i$ value determined in Paper II $\left(11.3 \mathrm{~km} \mathrm{~s}^{-1}\right)$. The veiling standard deviation was computed for each night using the veiling values obtained in the 6 separate orders. The rms deviation ranges from 0.04 to 0.14 , with a mean value of 0.07 over the 22 nights.

The derived veiling values are low, ranging from 0.2 to 0.7 , but are considerably higher than in Paper II, where they were all below 0.3 . A periodogram analysis suggests that the veiling varies periodically with a $8.2 \pm 0.3$ day period $(\mathrm{FAP}=0.22)$, which is consistent with the stellar rotation period given the much poorer time sampling of the spectroscopic data (see Fig. 4). The veiling is weak and almost constant in the photometric cycles where only a shallow brightness minimum is present, while it shows a strong peak near phase 0.4 in the deep minimum cycles. In Paper II, the veiling presented two maxima per photometric cycle, as if we were seeing 2 hot spots at the surface of the star, while in 2005 only one maximum per cycle is visible. We measured the equivalent width of the He I line by direct integration on the spectrum and computed line fluxes with the photometric measurements as $F(\mathrm{He} \mathrm{I})=$ const. $\times E W(\mathrm{He} \mathrm{I}) \times 10^{-0.4 m_{V}}$, 


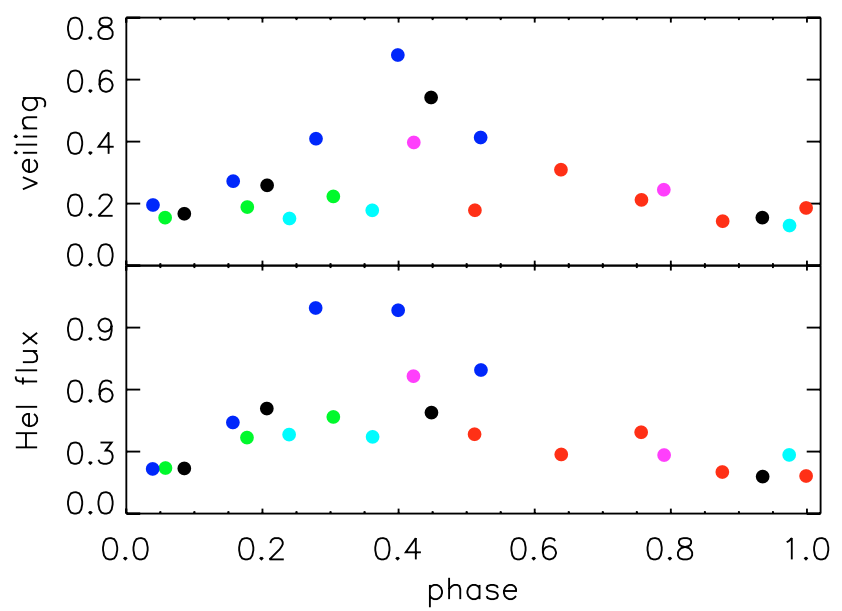

Fig. 4. Veiling (top) and He I line flux (bottom) variations folded in phase with a period of 8.22 days. The origin of phase is the same as for photometric measurements. Different cycles are shown by different colors (or shades of grey).

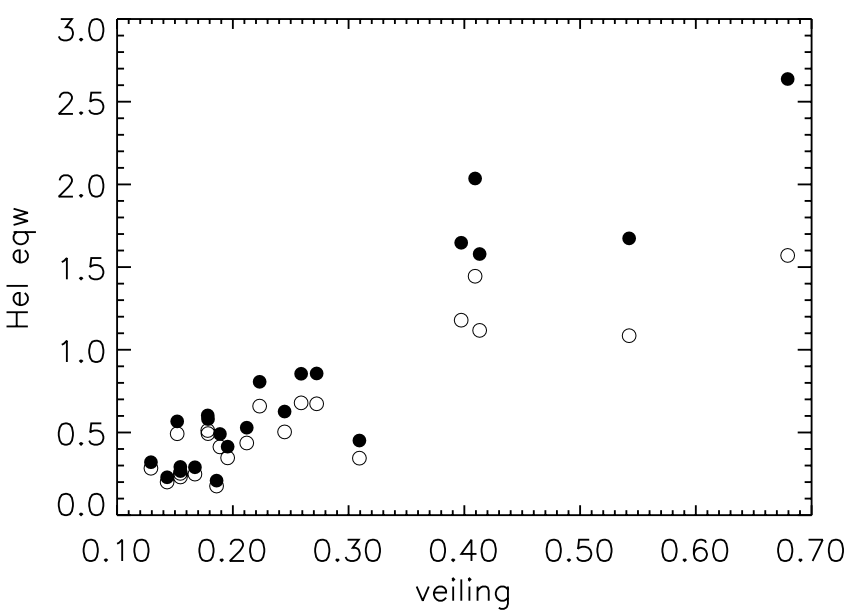

Fig. 5. He I equivalent width plotted against veiling. Filled (resp. empty) dots correspond to $E W(\mathrm{He}$ I) measurements on veiled (resp., deveiled) spectra.

where $m_{V}$ is interpolated from the light curve on the time of the spectroscopic observations. The rms error on the normalized He I flux is of order of 0.1 (cf. Fig. 4). We observe a strong correlation between the veiling and the He I line flux, as well as between veiling and $E W(\mathrm{He}$ I) measured either on the veiled or on the deveiled spectra (correlation coefficient of 0.9, cf. Fig. 5), and a weaker correlation between the veiling and $B-V$. The $B-V$ vs. veiling behavior is however still consistent with the 1999 results, lower $B-V$ values corresponding to higher veiling values.

Cross-correlation functions (CCFs) of the photospheric spectrum were automatically computed by the HARPS spectrograph reduction pipeline using a binary mask corresponding to a K5 template spectrum. CCFs provide high signal-to-noise information about the features present in the star's averaged photospheric spectrum. They were computed for each AA Tau's spectrum and are shown in Fig. 6. It is seen that the averaged photospheric line profiles are sometimes asymmetric and this could be the cause of the radial velocity variation noticed in Paper II.

We measured the radial velocities adjusting a Gaussian to the cross-correlation profiles in three different ways: fitting the profile centroid, the far wings, or an intermediate region between

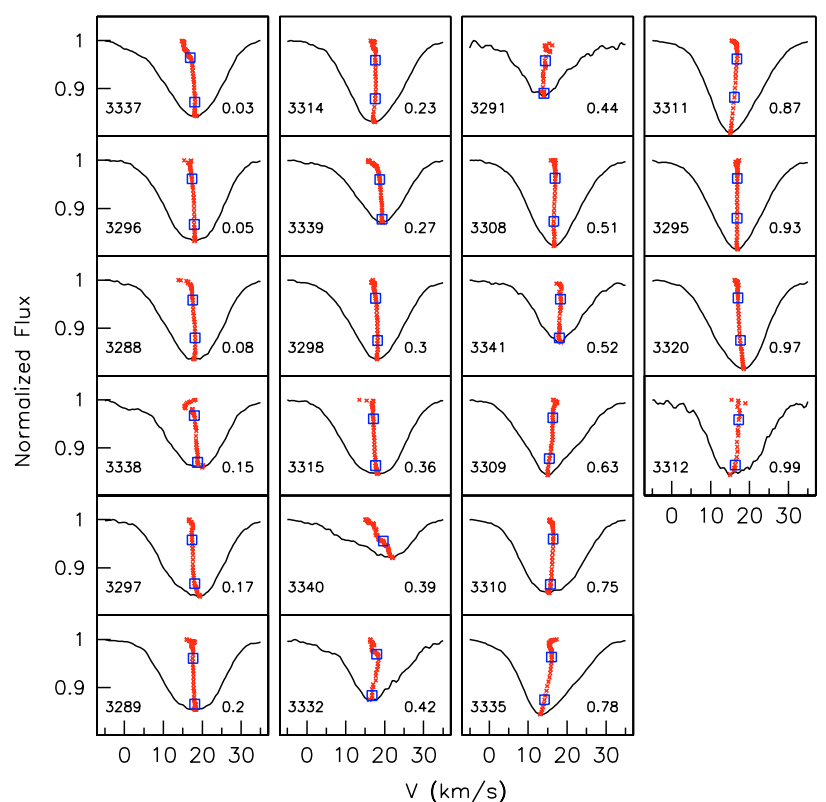

Fig. 6. Photospheric cross-correlation profiles (CCF). The profiles are ordered by increasing phase, as indicated in lower right corner of each panel, while Julian date is given in the lower left corner. The line bissector (cf. Queloz et al. 2001) is also shown (red crosses). The bissector average slope measures the degree of asymmetry of the CCF and is estimated as the velocity difference between the 2 data points indicated by open squares (see text), except for JD 3340 where the CCF is too shallow.

the minimum and the far wings. The mean radial velocity values obtained with the three different fitting procedures agree with each other $\left(v_{r}=17.3 \pm 1.6 \mathrm{~km} \mathrm{~s}^{-1}\right)$ and are consistent with the value obtained in Paper II $\left(v_{r}=17.1 \pm 0.9 \mathrm{~km} \mathrm{~s}^{-1}\right)$. Individual values can vary by as much as $1.5 \mathrm{~km} \mathrm{~s}^{-1}$ between different fits, but the three radial velocity data sets presented the same periodicity of $8.5 \pm 0.4$ days when submitted to a periodogram analysis (FAP $=0.02)$, which is slightly but not significantly different from the photometric period. All radial velocities below are expressed in the stellar rest frame $\left(V_{\mathrm{lsr}}\right)$.

In Fig. 7 we show the radial velocities obtained fitting the wings of the cross-correlation profiles, overplotted with the values determined in Paper II. The radial velocity variations exhibit the same period and about the same amplitude as those reported in Paper II. We cannot tell whether the phase is conserved over the 5 year timescale between the 2 epochs. This time span corresponds to about 220 periods and phase conservation could be tested only if the period was derived to an accuracy of $\simeq 0.1 \%$ or better. Nevertheless, the very good agreement between the two datasets suggests a similar origin for the observed radial velocity variations at the 2 epochs.

\subsubsection{Survey of emission line profiles}

A sample of AA Tau line profiles was presented in Paper II and the new observations show overall the same variety of emission profiles. For all lines except $\mathrm{H} \alpha$, we computed residual profiles by subtracting the continuum normalized and veiled spectra of V819 Tau from the continuum normalized spectra of AA Tau. In this process, we used the veiling value measured between $5425 \AA$ to $6660 \AA$, which exhibits no clear trend with wavelength. The veiling in the $\mathrm{H} \beta$ region is probably higher but AA Tau's veiling is in general small and the photospheric 


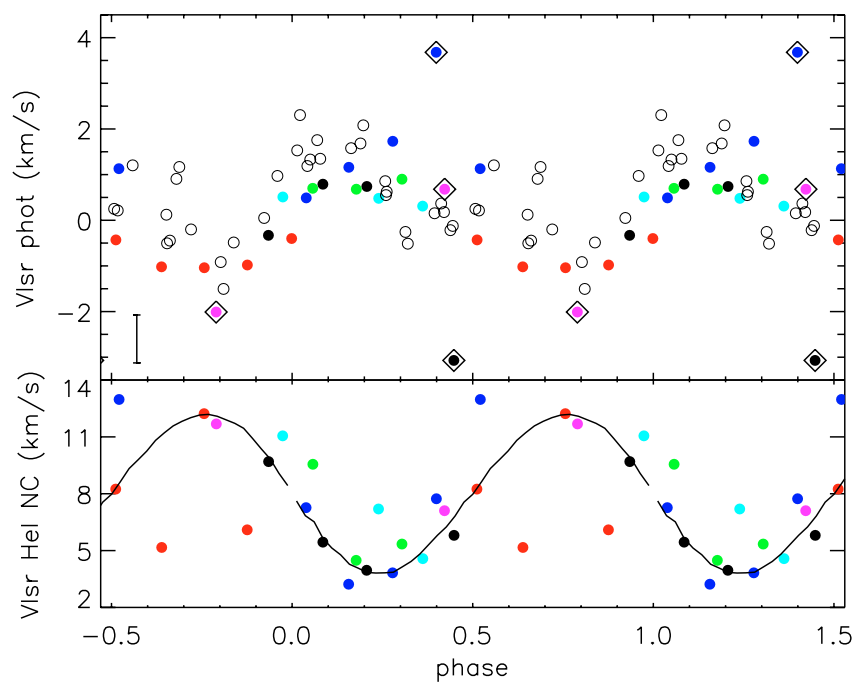

Fig. 7. Top panel: photospheric radial velocities as a function of phase. The open circles correspond to the values measured in the 1999 campaign (Paper II) and the filled symbols to the 2004 campaign. The phase of the 1999 points has been arbitrarily scaled to fit that of the 2004 dataset since the phase information is lost between the 2 epochs (see text). The points with an overplotted diamond correspond to very asymmetric cross-correlation profiles. The error bar in the left corner represents the mean error of the 1999 values. Bottom panel: He I radial velocities. The solid line shows the radial velocity variations expected from a hot spot located at a latitude of $70^{\circ}$ on the stellar surface.

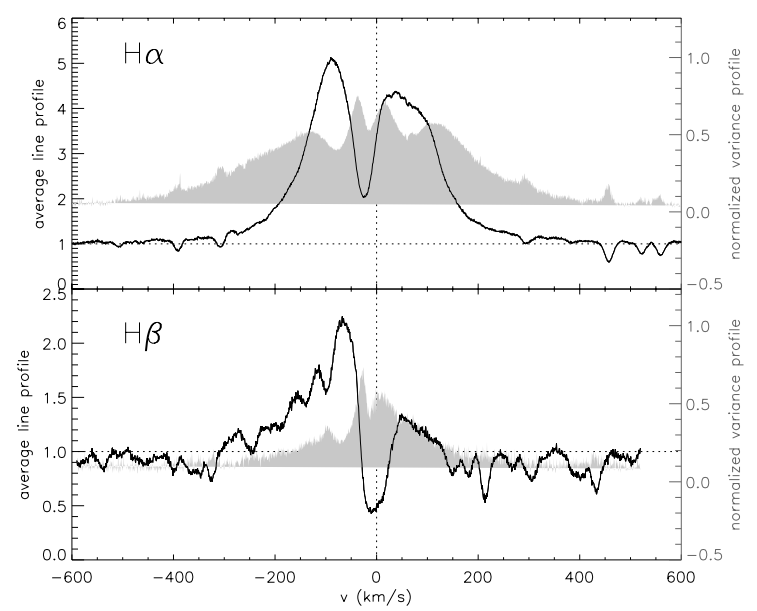

Fig. 8. Average lines profiles (solid line) and their variance (grey shaded area) are shown for $\mathrm{H} \alpha$ (top) and $\mathrm{H} \beta$ (bottom).

features are reasonably well removed from the $\mathrm{H} \beta$ region when we use a single veiling value.

In Fig. 8 we show the average $\mathrm{H} \alpha$ and $\mathrm{H} \beta$ profiles as well as their variance (see Johns \& Basri 1995). The average $\mathrm{H} \alpha$ profile and its variance observed during this new campaign are quite similar to those reported for the 1999 campaign (Paper II). This suggests that both the emission line region(s) and the intrinsic source(s) of variability remained the same over the years. The $\mathrm{H} \alpha$ average profile is reminiscent of the model $\mathrm{H} \alpha$ profile computed by Kurosawa et al. (2006) which assumes that the line is formed partly in an axisymmetric magnetospheric funnel flow and partly in a slowly accelerating disk wind both seen at a high inclination (see their Fig. A1). The mean $\mathrm{H} \beta$ profile, with a triangular blue wing, a central absorption and a secondary peak in the red wing, closely ressembles the $\mathrm{Pa} \beta$ profile computed by Kurosawa et al. (2005) as arising from an axisymmetric accretion funnel flow seen at high inclination (cf. their Fig. 9). This is consistent with the high inclination viewing angle to AA Tau $\left(i \sim 75^{\circ}\right.$, Paper I). The variance $\mathrm{H} \alpha$ profile also shares some similarity with variance profiles computed from models in which the accretion flow is confined to azimuthal curtains instead of being axisymmetric (Symington et al. 2005b), specifically a primary peak of variance close to zero velocity and high velocity secondary maxima in the blue and red wings. We note however that the degree of variability observed in the red wing of AA Tau's $\mathrm{H} \alpha$ profile is much larger than predicted by azimuthally confined curtain models.

In Figs. 9 and 10 we show the $\mathrm{H} \alpha$ and $\mathrm{H} \beta$ emission lines as a function of rotational phase. The deep, blueshifted central absorption is seen at all times, which presumably arises from a cold axisymmetric inner disk wind. The broad emission wings are also always present, which suggests a globally axisymmetric accretion flow onto the star to first order. However, we notice that when a deep minimum occurs in the light curve and the photosphere is obscured by the inner disk warp, a high velocity redshifted absorption component of $\mathrm{H} \alpha$ is clearly seen, with maximum strength near phase 0.5 . This suggests that we are observing downwards along the main accretion funnel flow at this phase. The redshifted absorption by the main accretion stream is even more dramatic in $\mathrm{H} \beta$ where the entire red emission is suppressed at phases near the photometric minimum and the redshifted absorption is sometimes observed below the continuum (like JD 3291, 3332, 3340). The cycles that only present a shallow photometric minimum do not show such a pronounced redshifted absorption component in the $\mathrm{H} \alpha$ lines (e.g. JD 3308). This is consistent with the low veiling and low He I equivalent width values (Fig. 4) in that case, all of which indicate that accretion is at a very low level when the obscuring circumstellar material is absent.

We decomposed the $\mathrm{H} \alpha$ profile using three Gaussians, corresponding to a centered emission, a blueshifted absorption and a redshifted absorption. The decomposition is not always straightforward (see Fig. 14 of Paper II) but the radial velocities of the three components are well determined by the Gaussian fits. We had noticed in Paper II a tight correlation between the radial velocity of the blueshifted (wind) and redshifted (accretion) absorption components in the $\mathrm{H} \alpha$ line that we interpreted as being due to the inflation of the stellar magnetosphere, caused by differential rotation between the stellar magnetic field lines and the inner disk. Such a correlation persists in the current data and the new values agree very well with the measurements of Paper II as can be seen in Fig. 11.

The He I line presents a narrow emission component (NC) that dominates the profile and half of the time a broad emission component (BC) as well. We then decomposed the He I line using one or two Gaussian emissions as shown in Fig. 12. The $\mathrm{NC}$ is relatively stable kinematically, with radial velocity measurements going from $+4 \mathrm{~km} \mathrm{~s}^{-1}$ to $+12 \mathrm{~km} \mathrm{~s}^{-1}$ and a $F W H M$ varying from $16 \mathrm{~km} \mathrm{~s}^{-1}$ to $20 \mathrm{~km} \mathrm{~s}^{-1}$. A periodogram analysis of the radial velocity variations suggests a possible period of $\sim 8.0 \pm 0.4$ days $(\mathrm{FAP}=0.30$ ), consistent with the 8.2-day photometric period (cf. Fig. 7). The equivalent width and the line flux of the NC vary considerably in the same period and are well correlated with the veiling variations. According to Beristain et al. (2001) the NC is formed near the accretion shock in the postshock region, and should strongly correlate with the veiling, as observed in AA Tau. The BC is much more kinematically variable than the $\mathrm{NC}$, with radial velocity values going from $-33 \mathrm{~km} \mathrm{~s}^{-1}$ to $+31 \mathrm{~km} \mathrm{~s}^{-1}$ and $F W H M$ ranging from $30 \mathrm{~km} \mathrm{~s}^{-1}$ to $40 \mathrm{~km} \mathrm{~s}^{-1}$. It is most of the time redshifted but was observed 

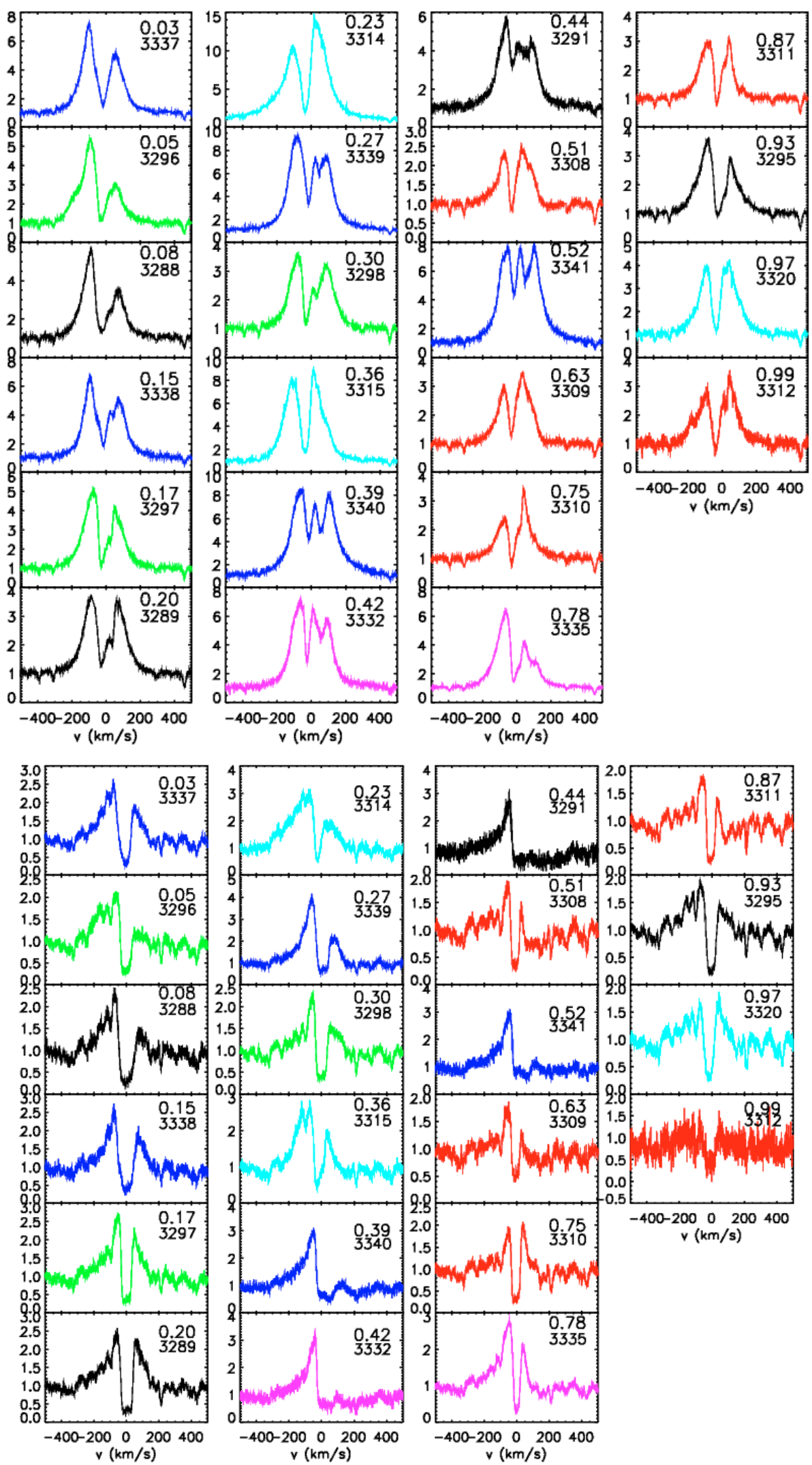

blueshifted twice (JD 3314 and 3315, cf. Fig. 12). According to Beristain et al. (2001) the BC could be either formed in the accretion column (if redshifted in excess of $8 \mathrm{~km} \mathrm{~s}^{-1}$ ) or in an accretion-driven hot stellar wind (if blueshifted in excess of $-30 \mathrm{~km} \mathrm{~s}^{-1}$ ). Our results show that a single star can move from
Fig. 9. $\mathrm{H} \alpha$ emission line profiles ordered by rotational phase (top panel number). Julian date is given (bottom panel number). Note the appearance of a redshifted absorption component from phase 0.39 to 0.52 .

Fig. 10. $\mathrm{H} \beta$ emission line profiles ordered by rotational phase (top panel number). Julian date is also given (bottom panel number). Note the appearance of a redshifted absorption component from phase 0.39 to 0.52 . the accretion dominated $\mathrm{BC}$ (redshifted) to a wind dominated BC (blueshifted) and back in a short period of time (a couple of nights).

In Fig. 13 we show our period search result for the $\mathrm{H} \alpha$ and He I lines. Periodograms were computed in each velocity 


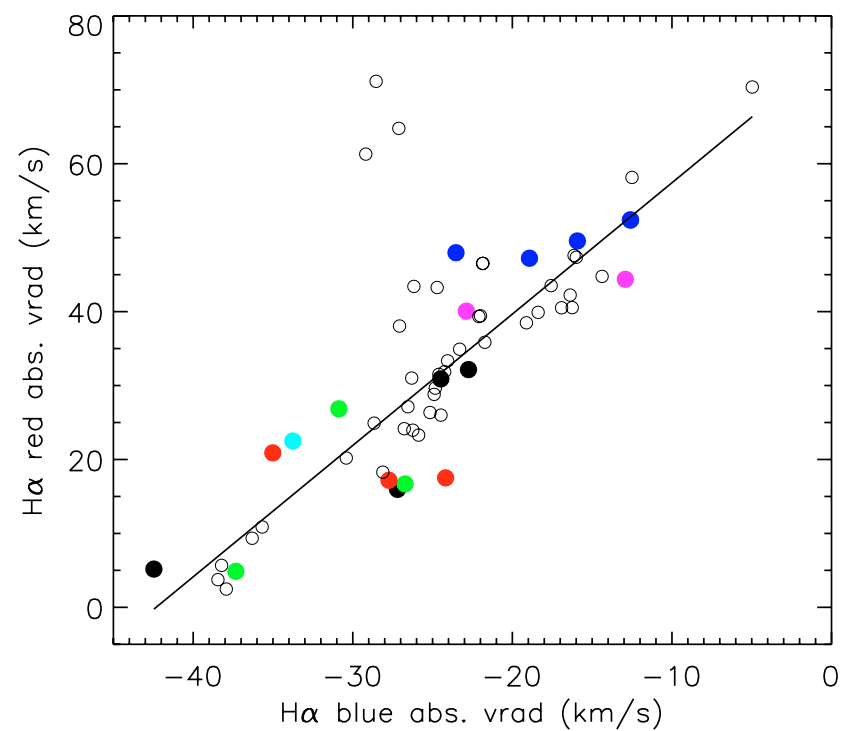

Fig. 11. Correlation between the radial velocity of the blueshifted (wind) and redshifted (accretion) absorption component in the $\mathrm{H} \alpha$ line (color data points: 2004, open data points: 1999).

channel on the intensity of the deveiled and normalized line profiles. Similar results are obtained from the analysis of veiled profiles. All the main emission lines show periodical intensity variations around $8.0 \pm 0.4$ days, consistent with the more accurately defined photometric period of 8.2 days. The maximum power in the 2D periodogram of the $\mathrm{H} \alpha$ (resp. He I) line occurs at $V_{\mathrm{lsr}} \simeq-10 \mathrm{~km} \mathrm{~s}^{-1}$ (resp. $3 \mathrm{~km} \mathrm{~s}^{-1}$ ) and corresponds to a FAP of 0.15 (resp. 0.10). This is the first time we find clear evidence for the emission line flux being modulated with the same period as the stellar flux (see Paper II). Most of the emission lines also show more marginal evidence for periodicities at 6.7 days.

\section{Discussion}

The new dataset obtained on AA Tau provides new and clear evidence for magnetospheric accretion in this object. We thus discuss in this section the observed variability pattern of the various diagnostics in the framework of the magnetically-channelled accretion concept, starting form the inner disk edge down to the stellar surface. In the following discussion, the origin of rotational phases $(\Phi=0)$ is taken as being the epoch of maximum continuum flux in the $V$-band. This maximum brightness level has proved very stable over at least the last 10 years (see Papers I, II) and likely corresponds to the unobscured photosphere. In addition, the veiling is low at this phase, so that the observed continuum flux is probably close to the star's photospheric flux (reddened by $A_{v}=0.8 \mathrm{mag}$, cf. Paper I).

\subsection{Characterizing the magnetospheric accretion/ejection region}

As the system rotates with a period of $8.22 \mathrm{~d}$, the continuum flux decreases to reach a minimum around $\Phi \simeq 0.5$. This behaviour had already been reported in previous campaigns, and we argued that the dimming of the system results from the central star being partly occulted by circumstellar material (Papers I, II). A supporting argument comes from the measurement of an increased polarization level during the eclipses (Ménard et al. 2003; see also O'Sullivan et al. 2005). We further argued that the optically thick occulting screen corresponds to the magnetically-warped,
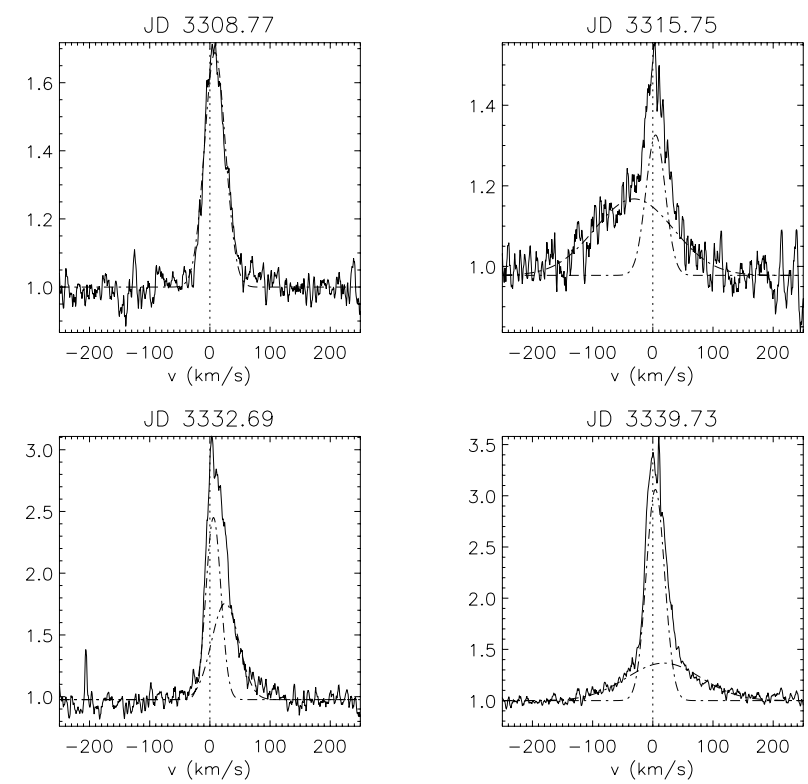

Fig. 12. He I line decomposition. The line profile is fitted by either one Gaussian when only a narrow component is present or two when a broad component appears.

dusty inner disk edge. As the periodicity of the eclipses is similar to the rotational period of the star, this locates the inner dust disk edge close to the corotation radius which, assuming Keplerian rotation, is $r_{\text {co }} \simeq 8.8 R_{\star}$.

The new dataset confirms both the periodic nature of the eclipses and their changing amplitude on week timescales (see Paper II). This can be seen in Figs. 2 and 3. Over nearly 170 days, corresponding to more than 20 rotations, the phase of the brightness minimum is conserved. While the depth of most eclipses is of order of $1 \mathrm{mag}$, we observed shallower eclipses with an amplitude of only a few 0.1 mag during 3 consecutive rotation cycles, located in the middle of the observing campaign (see Fig. 1). We conclude that the shape of the occulting screen can change abrutply on a timescale of days, leading to shallower eclipses for a few rotations, and is thereafter restored with nearly the same structure. A similar behaviour had been reported in Paper II for one rotation.

AA Tau's new light curve exhibits a hint of an asymmetric shape around the minimum, with a steeper egress than ingress (Fig. 3). At a given brightness level, the system also appears redder during ingress than during egress (Fig. 2). The shape of the eclipse reflects the azimuthal distribution of optically thick dust in the corotating inner disk warp. Such a slight light curve asymmetry, with a shallow decrease and a steep rise, may indicate a correspondingly asymmetric azimuthal distribution of dust at the inner disk edge, as qualitatively predicted by 3D numerical simulations of disk accretion onto an inclined dipole (Romanova et al. 2003).

If the warp of the inner disk edge results from its interaction with the stellar magnetosphere, as argued in Paper I, the dominant accretion funnel flows ought to face the observer close to the middle of the occultation. The analysis of the Balmer line profiles supports this view. Figures 9 and 10 clearly reveal the development of high velocity redshifted components in the profiles of both lines (also seen in $\mathrm{H} \gamma$ ) from $\Phi=0.39$ to $\Phi=0.63$, i.e., around the center of the eclipse. High velocity redshifted components are expected to be seen when the observer looks downwards the accretion funnel flow against the accretion shock (Hartmann et al. 1994). The line profiles thus indicate that the 


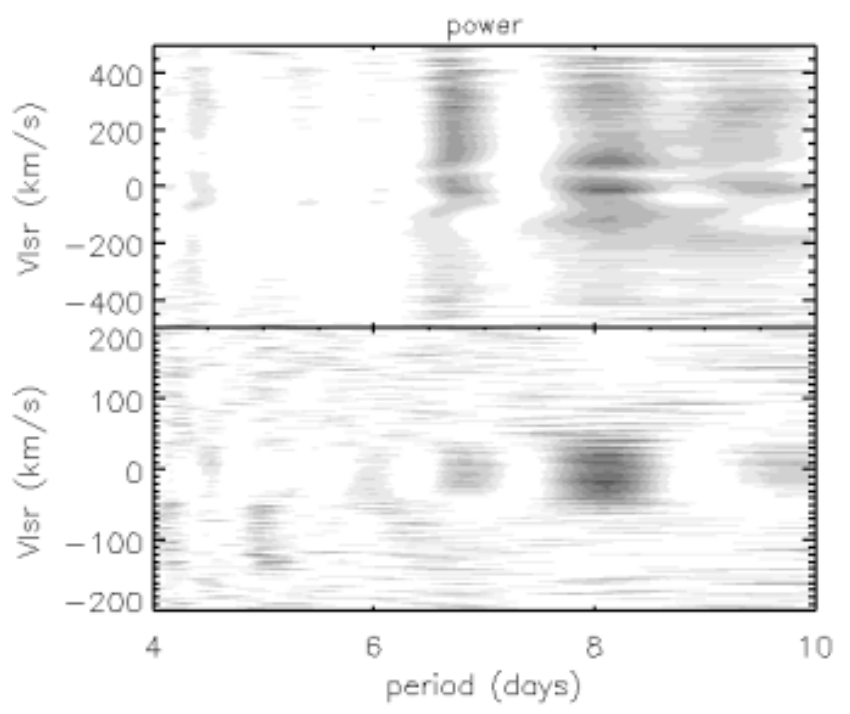

Fig. 13. Period search results of the $\mathrm{H} \alpha$ (top) and He I (bottom) lines. The power scale ranges from 0 (white) to a maximum value of about 6.0 (black)

center of the eclipse also corresponds to the time at which the accretion shock faces the observer. This strongly suggests that the inner disk warp is magnetically-driven indeed (Terquem \& Papaloizou 2000, Lai 1999).

The line profiles observed during one of the shallower eclipses (JD 3308, $\Phi=0.51$ ) exhibit a much shallower redshifted component than the profiles observed at similar phases during deep minima (e.g. JD 3341, $\Phi=0.52$, see Figs. 9 and 10). As the veiling was also measured to be weaker during shallow eclipses (see Fig. 4), this suggests that the accretion rate onto the star was smaller at that time. Hence, there seems to be a clear link between the structure of the occulting warp at the inner disk edge and the accretion rate onto the star, both presumably depending on the time variable magnetic configuration at the disk truncation radius. In addition, blueshifted $\mathrm{He}$ I broad emission components were observed only during one of the shallower eclipses (JD 3314, 3315), which suggests that a hot wind develops as accretion is depressed onto the star. Finally, the $\mathrm{H} \beta$ line profile reveals transient but significant redshited absorption components at phases opposite to the deep minima (e.g. JD 3295, $\Phi=0.05$ ). We speculate that these transient absorptions may be related to the viewing of funnel flows against the accretion shock located at the opposite magnetic pole on the stellar surface $(\Phi \simeq 0$, see Fig. 10 in Paper I).

The visibility of the hot spot at the stellar surface is measured by the variations of the He I line flux originating in the accretion shock at the bottom of the accretion funnel (Beristain et al. 2001). The He I line flux, usually dominated by a narrow component (Fig. 12), is modulated with the same $8.2 \mathrm{~d}$ period as the other diagnostics (Fig. 13) and shows a low level modulation of about a factor of 2, with a maximum around $\Phi=0.4$ (Fig. 4). The veiling is modulated in the same way and also peaks slightly before the center of the eclipse (Fig. 4). The close temporal coincidence between the maximum intensity of accretion shock diagnostics $(\Phi \simeq 0.3-0.6)$, the appearance of high velocity redshifted absorptions in Balmer line profiles $(\Phi=0.39-0.63)$, and the occurence of photometric eclipses $(\Phi \simeq 0.3-0.8)$ strongly supports the view that material is being preferentially accreted from the warped inner disk edge along magnetic field lines down to the stellar surface.
In addition to the low level modulation, both the He I line flux and the veiling exhibit intrinsic variations of larger amplitude on a shorter timescale. This is examplified by the 4 highest He I flux values in Fig. 4 and the corresponding measurements of veiling in Fig. 4. These episodes occur during the eclipses $(\Phi=$ $0.3-0.5)$. Whether these are transient episodes on a timescale of a few hours, such as accretion bursts or opacity changes in the occultation screen as previously reported in Paper I, or a slightly varying accretion rate onto the star from one cycle to the next cannot be easily decided from our dataset.

\subsection{The origin of periodic radial velocity variations}

One puzzling aspect of AA Tau's behaviour is the periodic radial velocity variations of the photospheric spectrum. We had reported this result in Paper II and offered possible interpretations, such as a planetary mass companion orbiting the star or the presence of a large cold spot at the stellar surface, none of which seemed satisfactory. The new dataset provides additional evidence for the modulation of the radial velocity of the photospheric spectrum with a period of $8.2 \mathrm{~d}$ and an amplitude of a few $\mathrm{km} \mathrm{s}^{-1}$, similar to what we previously reported (see Fig. 7). The source of radial velocity variations thus appears to be the same at the 2 epochs. The coincidence between the period of the radial velocity variations and the rotational period of the star strongly suggests the former results from spot modulation. Also, the amplitude of the radial velocity variations $\left(\sim 3 \mathrm{~km} \mathrm{~s}^{-1}\right)$ is a fraction of the star's rotational velocity $\left(v \sin i=11.3 \mathrm{~km} \mathrm{~s}^{-1}\right)$, consistent with spot modulation.

The high resolution spectral series obtained during the new campaign supports this view. The extrema of the radial velocity curve occur around rotational phases $0.2-0.3$ (maximum) and $0.7-0.8$ (minimum), while the star's rest velocity is measured around phases 0.0 and 0.5 , i.e. at maximum and minimum brightness (cf. Fig. 7). We argued above from the line profile analysis that the hot accretion shock faces the observer around $\Phi=0.5$. Assuming the hot spot is responsible for the modulation of the photospheric line profiles, one would expect to measure the star's rest velocity when the spot faces the observer $(\Phi=0.5)$ as well as at the opposite phase $(\Phi=0)$, while the largest $V_{\text {rad }}$ excursions ought to occur when the hot spot is close to the stellar limb $(\Phi \simeq 0.25$ and 0.75$)$. This is precisely what the phased radial velocity curve indicates, with $V_{\mathrm{lsr}} \sim$ $+1 \mathrm{~km} \mathrm{~s}^{-1}$ and $-1 \mathrm{~km} \mathrm{~s}^{-1}$ at $\Phi \simeq 0.25$ and 0.75 , respectively. In addition, the radial velocity of the He I narrow component (NC) also appears to be modulated, though with a larger scatter. It varies between $\sim+4 \mathrm{~km} \mathrm{~s}^{-1}$ and $\sim+12 \mathrm{~km} \mathrm{~s}^{-1}$ around $\Phi=0.25$ and $\Phi=0.75$, respectively, i.e. with a phase opposite to that of the photospheric radial velocity variations, as expected from hot spot modulation. The time averaged velocity of the He I line $\mathrm{NC}\left(V_{\mathrm{lsr}} \sim 8 \mathrm{~km} \mathrm{~s}^{-1}\right)$ presumably reflects the postshock velocity at the magnetospheric footpoint on the stellar surface (Beristain et al. 2001).

Figure 7 also shows that a few $V_{\text {rad }}$ measurements strongly depart from the low level periodic $V_{\text {rad }}$ variations. These measurements where obtained during photometric minima $(\Phi=$ $0.39,0.42,0.44$; JD 3340, 3332, 3291) at a time when the veiling was also the highest. The corresponding correlation profiles (CCFs) are extremely asymmetric (cf. Fig. 6) and such asymmetries may conceivably alter the accuracy of the $V_{\text {rad }}$ measurements. CCFs obtained outside theses phases show some level of asymmetry as well, but usually not as pronounced, as expected from the modulation by a hot surface spot (e.g. Petrov et al. 2001). The largest CCF asymmetries observed during the 


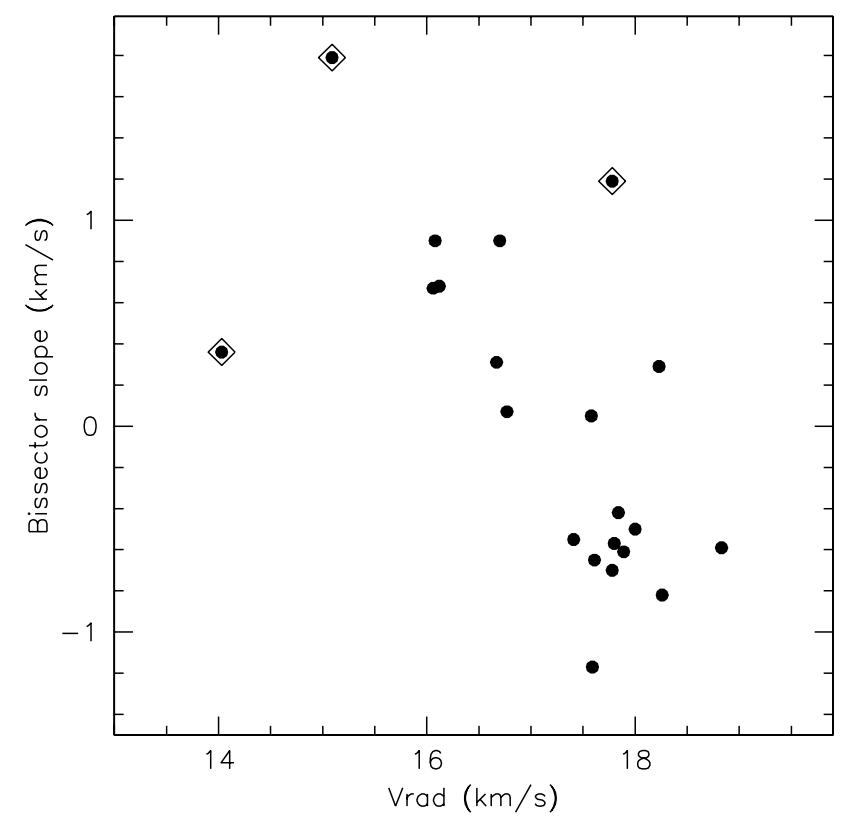

Fig. 14. The CCF bissector "slope" is plotted as a function of the measured photospheric radial velocity. The points with an overplotted diamond correspond to very asymmetric CCFs. The inverse correlation seen between the 2 quantities indicates that radial velocity variations mostly result from spots at the stellar surface.

deep photometric minima suggest that the extreme $V_{\text {rad }}$ excursions are related to the partial occultation of the central star by the asymmetric inner disk warp, and are superimposed onto the lower level $V_{\text {rad }}$ modulation by the hot spot. Note that only few measurements strongly depart from the hot spot $V_{\text {rad }}$ modulation, which suggests that the azimuthal structure of the inner disk warp occulting the star is relatively smooth, as also suggested by the shape of the photometric eclipses.

Hence, the modulation of the photospheric radial velocity in AA Tau's most likely results from an inhomogeneous brightness distribution on the stellar surface. Its phase is consistent both with the visibility of the hot accretion spot derived above from veiling and He I flux measurements, and with the He I line radial velocity variations. For a hot spot occupying a few percent of the stellar photosphere, the radial velocity amplitude of the He I line is related to the latitude $\theta$ of the accretion shock at the stellar surface by: $\Delta V_{\mathrm{rad}}=2 v \sin i \cdot \cos \theta$. With $\Delta V_{\mathrm{rad}} \simeq 8 \mathrm{~km} \mathrm{~s}^{-1}$ and $v \sin i=11.3 \mathrm{~km} \mathrm{~s}^{-1}$, we derive $\theta \simeq 70^{\circ}$. The solid line in Fig. 7 shows the expected He I radial velocity variations from a model featuring a small hot spot located at a latitude of $70^{\circ}$. The model curve fits reasonably well the observed $\mathrm{He}$ I radial velocity variations, at least when deep eclipses are seen in the light curve. As the accretion shock is thought to occur at the feet of the funnel flows on the stellar surface close to the magnetic pole, we deduce that the axis of the large scale magnetosphere is tilted relative to the star's spin axis by about $20^{\circ}$. This is consistent with the $12^{\circ}$ tilt derived by Valenti \& Johns-Krull (2004) from spectropolarimetric measurements, assuming a stellar inclination of $66^{\circ}$. Note that the radial velocity of the He I line is modulated around a mean value of $V_{\mathrm{lsr}} \sim 8 \mathrm{~km} \mathrm{~s}^{-1}$, the putative post-shock velocity, and not around the photospheric velocity, as would be expected for a small single hot spot. This suggests a more complex structure for the accretion shock, such as a circumpolar ring around the magnetic axis (Mahdavi \& Kenyon 1998; Romanova et al. 2004).
The observed periodic radial velocity variations of absorption and emission lines can thus be, at least partly, accounted for by the presence of a hot spot at the stellar surface. Whether a hot spot alone fully accounts for the radial velocity curve of the photospheric spectrum is however unlikely. Firstly, we note that the amplitude of the $V_{\text {rad }}$ variations is about the same in 1999 and in 2003 (cf. Fig. 7) while the veiling was significantly higher at the latter epoch. If hot spots were responsible for the radial velocity excursions, one might expect the amplitude of the $V_{\text {rad }}$ curve to increase with the spots brightness, which is not seen here. Secondly, the line shape distorsion caused by a hot continuum spot is the same as that procuded by a cold spot (e.g. JohnsKrull \& Hatzes 1997). In Paper II, we found that a cool spot would have to cover about $50 \%$ of the stellar surface to produce the observed $V_{\text {rad }}$ variations. This is much larger than the expected size of AA Tau's accretion spots, of order of a few percent (Paper I). Hence, it is unlikely that a hot spot alone can account for the observed $V_{\text {rad }}$ amplitude. Additional sources of variations are probably required, such as the occulting effect of the warp inner disk and/or large cold spots clustered around the hot accretion spot at the stellar surface. We also note that similar radial velocity variations were found in the more active CTTS RW Aur and interpreted as resulting from the modulation by large chromospheric spots (Petrov et al. 2001). Detailed Doppler Imaging of the star would be needed to address this issue. Note however that these additional components do not affect the conclusion regarding the radial velocity variations of the He I NC, which more directly traces the accretion hot spot on the stellar surface.

A final indication that the $V_{\text {rad }}$ modulation primarily results from stellar spots is provided by the analysis of the line profile shape and its deformation as a function of rotational phase. Following the method outlined by Queloz et al. (2001), we computed the bissector of the photospheric cross-correlation function (CCF) at each phase (cf. Fig. 6). The bissector slope is seen to vary as a function of phase. The average slope is computed as the velocity difference between the upper and lower regions of the bissector, $V_{\mathrm{u}}-V_{\mathrm{l}}$, where $V_{\mathrm{u}}$ (resp. $V_{\mathrm{l}}$ ) is the median velocity of the bissector in the intensity range $0.93-0.98$ (resp. $0.80-0.85)$. The bissector slope is plotted as a function of the measured $V_{\text {rad }}$ in Fig. 14. A clear inverse correlation is seen between the bissector slope and $V_{\text {rad }}$, as expected for spot-induced $V_{\text {rad }}$ variations (Queloz et al. 2001). In contrast, no such correlation would exist if the periodic $V_{\text {rad }}$ variations were due to stellar oscillations or to the reflex motion of the star in response to an orbiting body (Dall et al. 2006). A few measurements strongly depart from the observed correlation, as already noticed above for $V_{\text {rad }}$ measurements (cf. Fig. 7), and may indicate the additional effect of the inner disk warp partly occulting the stellar photosphere at times of deep photometric minima.

\subsection{Non steady accretion on a week timescale}

On a timescale longer than the star's rotational period, we confirm the correlation we reported in Paper II between the radial velocity of the blueshifted (wind) and redshifted (accretion) absorption components of the $\mathrm{H} \alpha$ profile (see Fig. 11). In Paper II, we interpreted this correlation as "magnetospheric inflation cycles". Following dynamical models of magnetic star-disk interaction (e.g. Goodson \& Winglee 1999) accretion from the inner disk onto the star leads to the inflation of the magnetic funnel flows due to differential rotation between the inner disk edge and the stellar surface. Past some critical point, the field lines open and reconnect, then reducing the accretion flow onto the star and simultaneously leading to enhanced wind outflow. After field 
lines have reconnected, the initial magnetic dipolar configuration is restored and a new cycle starts. We argued in Paper II that the projected radial velocity of the redshifted absorption components of the $\mathrm{H} \alpha$ line measure the curvature of the accretion funnel flow : as the magnetosphere inflates, the projected radial velocity of the redshifted component decreases (see Fig. 19 in Paper II). If the collimated wind originates at or close to the disk inner edge, the projected radial velocity of the blueshifted absorption component simultaneously increases, thus yielding the observed correlation. Note that this simple geometric interpretation favors inner disk winds (e.g. Alencar et al. 2005) over accretion driven stellar winds (e.g. Matt \& Pudritz 2005).

The results of the new campaign tend to confirm this interpretation. Just before and during the episode of shallow eclipses and depressed accretion onto the star (JD 3305-3330), the projected radial velocity of the accretion flow is small (from +5 to $+30 \mathrm{~km} \mathrm{~s}^{-1}$ ) and that of the inner disk wind is large (from -45 to $-25 \mathrm{~km} \mathrm{~s}^{-1}$ ). According to the above interpretation, this would correspond to a phase of field line inflation and opening. The lower accretion rate onto the star is also accompanied by the development of a hot wind outflow as traced by the appearence of a broad blueshifted component in the He I profile. Just after this episode, as deep eclipses and enhanced accretion resume, the projected radial velocity of the accretion flow is larger (from +40 to $+50 \mathrm{~km} \mathrm{~s}^{-1}$ ) and that of the inner disk wind is smaller (from -25 to $-10 \mathrm{~km} \mathrm{~s}^{-1}$ ), as expected if the initial dipolar magnetospheric configuration has been restored at this point. The observed timescale for the disappearance and restoration of deep eclipses is of order of a week (see Fig. 1). The dissipation timescale of the inner disk warp through bending waves, $\tau_{\mathrm{w}}$, in response to the disturbance of the underlying magnetic configuration, is given by $\tau_{\mathrm{w}}=2 r_{\mathrm{c}} / c_{\mathrm{s}}=2 r_{\mathrm{c}} /(\Omega \cdot h)$, where $r_{\mathrm{c}}$ is the disk truncation radius, $c_{\mathrm{s}}$ is the local sound speed, $\Omega$ the Keplerian velocity, and $h$ the warp thickness (Terquem, priv. comm.). At a distance $r_{\mathrm{c}}=8.8 R_{\star}$ and for a warp vertical thickness $h / r_{\mathrm{c}} \sim 0.3$ (cf. Paper I), this yields $\tau_{\mathrm{w}} \sim 4$ days, which is consistent with the observations.

The variations of the accretion and outflow diagnostics on a timescale of several rotation periods in AA Tau can thus be consistently understood as resulting from episodes of strong accretion onto the star together with reduced or absent hot wind (whenever the dipolar magnetosphere allows the disk material to fall onto the star) alternating with episodes of reduced accretion and enhanced hot wind possibly driven by magnetic reconnections (when the magnetosphere inflates due to differential rotation), with the whole process occurring on a timescale of a few weeks. In addition, the stability of the deep central absorption in the Balmer line profile suggests that a cold inner disk wind is always present. These results tend to support the concept of magnetospheric inflation cycles on a timescale of several rotation periods in accreting $\mathrm{T}$ Tauri stars, although the periodic character of such cycles as predicted by dynamical models remains to be established from longer time series.

\subsection{AA Tau's long term photometric behaviour}

Finally, Fig. 15 offers a long term view of AA Tau's photometric behaviour, as recorded in the last 10 years. The shape and depth of the eclipses do vary on a timescale of weeks within each observing season, and small phase changes (up to 0.1-0.2) occur as well on this time scale. Nevertheless, the overall pattern of photometric variability remains globally stable within each season over a timescale of several months (Fig. 3). Figure 15 indicates that the long term (years) evolution of the inner disk structure

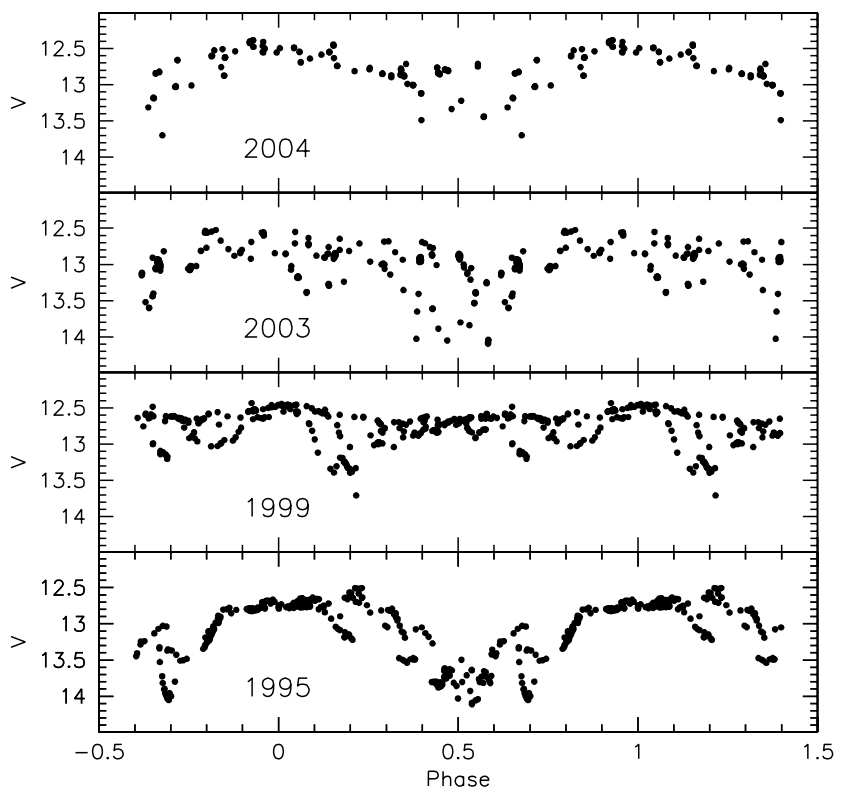

Fig. 15. $V$-band light curves of AA Tau at different epochs: 1995 (Paper I), 1999 (Paper II), 2003 (unpublished), 2004 (this paper). All light curves have been folded in phase with the same period of $8.22 \mathrm{~d}$ but the origin of phase for each season is arbitrary. Note how the shape and depth of the eclipses vary on a timescale of several years.

can be more drastic. The set of light curves obtained at different epochs $(1995,1999,2003,2004)$ exhibit quite different patterns. In 1995, a broad and deep eclipse was seen per rotation. In 1999, 2 shallower eclipses were observed per cycle. The 2003 light curve exhibits a clear primary eclipse, whose amplitude varies, and may still bear evidence for a shallow secondary eclipse. The 2004 light curve reported here displays only one eclipse per cycle, broader and shallower than observed in 1995. The changing pattern of the eclipses over the years reflects the varying structure of the inner disk warp occulting the star. This suggests that the large scale component of the stellar magnetic field interacting with the inner disk and producing the warp can significantly vary over the years.

\section{Conclusions}

We have analyzed a long time series of high resolution spectra and simultaneous photometry obtained for the accreting T Tauri star AA Tau. The photometric diagnostics provide clues to the structure of the dusty inner disk edge interacting with the star's magnetosphere while various spectral diagnostics probe the accretion funnel flows onto the star as well as the accretion shock at the stellar surface. To first order, the observed variability of these diagnostics on a timescale ranging from days to months supports the view of time-dependent, magnetically-controlled accretion from the inner disk edge onto the star.

The stellar magnetosphere disrupts the inner disk at the corotation radius, located about $9 R_{\star}$ above the photosphere. At the disk truncation radius, material is lifted away from the disk plane by the inclined stellar magnetosphere which results in an asymmetric dusty warp at the disk inner edge. The inner disk warp corotates with the star and partly occults the stellar photosphere periodically $(P=8.22 \mathrm{~d}$ ), yielding the deep and broad periodic eclipses observed in the light curve. As the star is periodically 
occulted, accretion diagnostics are the strongest (veiling, He I flux), which indicates that the accretion shock at the stellar surface is located at the same rotational phase as the inner disk warp. High velocity redshifted absorption components also appear in the Balmer line profiles at this rotational phase, being formed in the main accretion flow which connects the inner disk to the accretion shock. Hence, from the temporal coincidence of various photospheric and spectral diagnostics, we are able to demonstrate for the first time the spatial continuity between the inner disk warp, the main accretion funnel flow, and the accretion shock at the stellar surface, as qualitatively expected from models in which accretion from the inner disk onto the star is mediated by a large-scale inclined magnetosphere. The line variability is thus modulated by the corotation of the asymmetric magnetospheric funnel flow onto the star. Nevertheless, some components of the emission line profiles remains fairly stable, which suggests that the degree of asymmetry of the magnetospheric structure and of the inner disk wind is moderate. By modelling the radial velocity variations of the star and of the accretion shock, we derive an inclination of $20^{\circ}$ between the axis of the large scale magnetosphere and the stellar rotational axis.

On a timescale of several rotation periods, we also find that the accretion rate onto the star varies, with episodes of strong accretion and reduced hot wind alternating with episodes of weaker accretion and stronger hot wind. As accretion onto the star weakens, the depth of the photometric eclipses is also significantly reduced. This is readily explained by the fact that the accretion flow onto the star and the disk inner warp are both driven by the underlying magnetic structure interacting with the disk. In other words, time-variable eclipses and accretion rate onto the star both reflect temporal variations in the topology of the magnetic field lines which connect the star to the inner disk. We find that the observed variability of accretion and wind diagnostics in the Balmer and $\mathrm{He}$ I line profiles on a timescale of weeks is consistent with "magnetospheric inflation", i.e., field line expansion resulting from differential rotation between the inner disk and the stellar surface. As the magnetic field lines inflate and eventually become open, the amount of disk material that can be loaded into closed field lines decreases, thus reducing the accretion rate onto the star, while more material can be driven away along open field lines, thus leading to an enhanced hot wind, possibly driven by magnetic reconnections close to the star. In addition, evidence for a cold inner disk wind is seen at all times. The timescale for the development of magnetospheric inflation is several (typically $\sim 5-10$ ) rotational periods.

Finally, the comparison of AA Tau's light curves obtained over a timescale of 10 years, between 1995 and 2004, indicates that, even though periodic eclipses are seen at all epochs on a rotational timescale, their shape and depth drastically varies on a timescale of years. This indicates that, in addition to transient perturbations of the magnetospheric structure occuring on a timescale of weeks ("magnetospheric inflation"), the overall magnetospheric structure does change quite substantially over the years. This large-scale changes may conceivably be related to long term variations in the disk accretion rate or be intrinsic to the stellar magnetic field such as, e.g., magnetic cycles.

Acknowledgements. We thank A. Pal, J. Benko, S. Csizmadia, Z. Kiss, A. Kospal, M. Racz, K. Sarneczky, and R. Szabo who took part in the observations obtained at Konkoly Observatory. We thank the referee, C. Johns-Krull, for insightful comments on the manuscript. S.H.P.A. acknowledges support from CNPq (grant 201228/2004-1), Fapemig and LAOG. Z. Balog received support from Hungarian OTKA Grants TS049872, T042509 and T049082.

\section{References}

Akeson, R. L., Boden, A. F., Monnier, J. D., et al. 2005, ApJ, 635, 1173

Alencar, S. H. P., Basri, G., Hartmann, L., \& Calvet, N. 2005, A\&A, 440, 595

Bacciotti, F., Ray, T. P., Mundt, R., Eislöffel, J., \& Solf, J. 2002, ApJ, 576, 222 Beristain, G., Edwards, S., \& Kwan, J. 2001, ApJ, 551, 1037

Basri, G., \& Bertout, C. 1989, ApJ, 341, 340

Bouvier, J., Chelli, A., Allain, S., et al. 1999, A\&A, 349, 619 (Paper I)

Bouvier, J., Grankin, K. N., Alencar, S. H. P., et al. 2003, A\&A, 409, 169 (Paper II)

Bouvier, J., Alencar, S. H. P., Harries, T. J., Johns-Krull, C. M., \& Romanova, M. M. 2007, Protostars and Planets V, ed. B. Reipurth, D. Jewitt, \& K. Keil, (University of Arizona Press: Tucson), 951, 479

Dall, T. H., Santos, N. C., Arentoft, T., Bedding, T. R., \& Kjeldsen, H. 2006, A\&A, 454, 341

Dougados, C., Cabrit, S., Lavalley, C., \& Ménard, F. 2000, A\&A, 357, L61

Dupree, A. K., Brickhouse, N. S., Smith, G. H., \& Strader, J. 2005, ApJ, 625, L131

Dworetsky, M. M. 1983, MNRAS, 203, 917

Edwards, S., Fischer, W., Kwan, J., Hillenbrand, L., \& Dupree A. K. 2003, ApJ, 599, L41

Goodson, A. P., \& Winglee, R. M. 1999, ApJ, 524, 159

Hartigan, P., Hartmann, L., Kenyon, S., Hewett, R., \& Stauffer, J. 1989, ApJS, 70,899

Hartmann, L., Hewett, R., \& Calvet, N. 1994, ApJ, 426, 669

Hartigan, P., Edwards, S., \& Ghandour, L. 1995, ApJ, 452, 736

Herbst, W., Bailer-Jones, C. A. L., Mundt, R., Meisenheimer, K., \& Wackermann, R. 2002, A\&A, 396, 513

Horne, J. H., \& Baliunas, S. L. 1986, ApJ, 302, 757

Johns, C. M., \& Basri, G. 1995, AJ, 109, 2800

Johns-Krull, C. M., \& Hatzes, A. P. 1997, ApJ 487, 896

Johns-Krull, C. M., Valenti, J. A., Hatzes, A. P., \& Kanaan, A. 1999, ApJ, 510, L41

Johns-Krull, C. M., Valenti, J. A., \& Saar, S. H. 2004, ApJ, 617, 1204

Koenigl, A. 1991, ApJ, 370, L39

Kurosawa, R., Harries, T. J., \& Symington, N. H. 2005, MNRAS, 358, 671

Kurosawa, R., Harries, T. J., \& Symington, N. H. 2006, MNRAS, 370, 580

Lai, D. 1999, ApJ, 524, 1030

Mahdavi, A., \& Kenyon, S. J. 1998, ApJ, 497, 342

Matt, S., \& Pudritz, R. E. 2005, ApJ, 632, L135

Mayor, M., Pepe, F., Queloz, D., et al. 2003, ESO Messenger, 114, 20

Ménard, F., \& Bertout, C. 1999, The Origin of Stars and Planetary Systems, NATO ASIC Proc. 540, 341

Ménard, F., Bouvier, J., Dougados, C., Melnikov, S. Y., \& Grankin, K. 2003, A\&A, 409, 163

O'Sullivan, M., Truss, M., Walker, C., et al. 2005, MNRAS, 358, 632

Petrov, P. P., Gahm, G. F., Gameiro, J. F., et al. 2001, A\&A, 369, 993

Pojmanski, G., Pilecki, B., \& Szczygiel, D. 2005, Acta Astronomica, 55, 275

Queloz, D., Henry, G. W., Sivan, J. P., et al. 2001, A\&A, 379, 279

Roberts, D. H., Lehar, J., \& Dreher, J. W. 1987, AJ, 93, 968

Romanova, M. M., Ustyugova, G. V., Koldoba, A. V., \& Lovelace, R. V. E. 2002, ApJ, 578, 420

Romanova, M. M., Ustyugova, G. V., Koldoba, A. V., Wick, J. V., \& Lovelace, R. V. E. 2003, ApJ, 595, 1009

Romanova, M. M., Ustyugova, G. V., Koldoba, A. V., \& Lovelace, R. V. E. 2004, ApJ, 610, 920

Shang, H., Glassgold, A. E., Shu, F. H., \& Lizano, S. 2002, ApJ, 564, 853

Shevchenko, V. S., Grankin, K. N., \& Ibragimov, M. A. 1991, Informational Bulletin on Variable Stars, 3652, 1

Symington, N. H., Harries, T. J., Kurosawa, R., \& Naylor, T. 2005a, MNRAS, 358,977

Symington, N. H., Harries, T. J., \& Kurosawa, R. 2005b, MNRAS, 356, 1489

Terquem, C., \& Papaloizou, J. C. B. 2000, A\&A, 360, 1031

Valenti, J. A., \& Johns-Krull, C. M. 2004, Ap\&SS, 292, 619

Vrba, F. J., Rydgren, A. E., Chugainov, P. F., Shakovskaia, N. I., \& Weaver, W. B. 1989, AJ, 97, 483 